\title{
Design and Experimental Analysis of Rail Type Electromagnetic Weapons Systems
}

\author{
A. KAKILLI ${ }^{a, *}, \dot{I}$. YILDIZ ${ }^{b}$ \\ ${ }^{a}$ Marmara University, Vocational School of Technical Sciences, Göztepe, Istanbul, Turkey \\ ${ }^{b}$ Artvin Coruh University, Hopa Vocational School, Artvin, Turkey
}

\begin{abstract}
In the study, electromagnetic weapon systems that are developed as an alternative to modern weapon systems are examined and a design for a rail type electromagnetic weapon system is intended. After a literature review on the subject, the comparison of electromagnetic and conventional weapons is made and models of electromagnetic weapons are elaborated. In the study, information of various electromagnetic weapons is provided but the focus is determined to be rail type electromagnetic weapons. Rail type electromagnetic weapons are examined in the study and the design of a low-power weapon prototype is made. For the model developed under laboratory conditions, 8 capacitors are used as a source of ignition. With the rail type electromagnetic weapon system designed, 2613.9 Joule energy is reached. The system components projected in theoretical calculations are enhanced throughout the application phase and are optimized for rail type weapon systems. For making theoretical studies and for drawing the graphs MATLAB software program is utilized. As a consequence, theoretical and empirical data is compared and commented, and some recommendations are formed.
\end{abstract}

DOI: 10.12693/APhysPolA.127.1150

PACS: 89.20.Dd, 03.50.De, 41.20-q

\section{Introduction}

The idea of magnetic weapon that was similar to a linear motor was first suggested in 1918 but could not succeed. In 1922 a French scientist Louis Octave obtained a patent for "Electric Apparatus for Propelling Projectiles" however, the project could not be accomplished [1].

During the 2nd World War, Germany revived the idea for antiaircraft weapons. Nevertheless, the necessary electrical power could not be supplied and therefore the idea had always been a theory. In 1950, the 500 Megajoule power plant constructed by an Australian physician Mark Oliphant proved that the idea of electromagnetic weapon is not just a theory. Today the endeavor for developing electromagnetic weapons is still in progress [2].

\section{Electromagnetic weapons}

In the literature electromagnetic weapons are examined under four groups, namely coil type, linear, rail type and hybrid type.

\subsection{Coil Type Electromagnetic Weapons}

Coil type electromagnetic weapons rests on the principle of launch of the object located between the solenoids with the electrical propelling force created through a series of solenoid coils. Coil type launchers play crucial roles for the launches of massive objects with mainly medium speeds [3].

A series of solenoid coils are sequentially connected to sources of stored electrical energy in order to create a magnetic field that moves in the barrel of the launcher.

${ }^{*}$ corresponding author; e-mail: kakilli@marmara.edu.tr
In a coil type launcher, a current should be applied to the solenoids as if the armature would emanate the solenoid. Various timing and detection circuits are described that are based on optical or inductive sensing of the armature position and fast semiconductor switches [4].

\subsection{Linear electromagnetic weapons}

Linear electromagnetic weapons, or linear induction launchers, are air-core solenoid launchers that work with the principle of asynchronous motor. By being supplied from a multiphase alternating current the solenoids at the barrel creates moving magnetic field in the barrel. In order to increase efficiency the barrel may be divided into chambers. Each part is supplied by distinct sources having distinct frequencies. The frequency in a part is more than the one in the previous part.

\subsection{Hybrid type electromagnetic weapons}

Hybrid type electromagnetic weapons are systems that involve both rail and coil type launchers in their structures. The design and construction of hybrid launchers are much more difficult than rail or coil type launchers. The superiority of hybrid type launchers compared to rail type launchers that necessitate high current or coil type launchers that can be constructed with high costs is that they need lower current in order to attain the same results.

\subsection{Rail type electromagnetic weapons}

Rail type electromagnetic weapons are based on the principle of launching of an object placed between two metal rails with electrical repelling force created. A simple rail type electromagnetic weapon basically comprises of three parts. These are; conductive rails that provide the formation of magnetic field, conductive projectile that complete the circuit, and the power plant that supply the necessary voltage to the rails [5]. 
Electromagnetic weapons have many advantages compared to gunpowder weapons. Launching the projectiles with high speeds by using energy directly is one of the most important advantages. With a large kinetic energy these weapons can make long-range hypersonic shots in a short time. Today test shots are realized at the speed of $9 \mathrm{~km} / \mathrm{s}$. For the time being, the probable maximum speed of electromagnetic weapons is accepted to be $140 \mathrm{~km} / \mathrm{s}$ with the construction of high-tech weapons and this figure is much faster than today's gunpowder weapons hav-

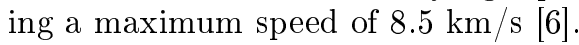

\section{Calculations for electromagnetic weapon}

Lorentz force and Biot-Savart law are utilized while constructing electromagnetic weapons. Under the electromagnetic weapon technology there is the interaction of current and magnetic field. This interaction is known as the Lorentz force and is defined with the following Equation [7, 8].

$$
\boldsymbol{F}=q\left(\boldsymbol{v}_{\boldsymbol{d}} \times \boldsymbol{B}\right) .
$$

Here $F$ denotes force; $q$, load; $v_{d}$, drift velocity; and $B$ denotes the magnetic field formed between the rails. According to Biot-Savart law, the current at the rails creates a magnetic field between the rails. Then, the current pass through the projectile and a magnetic field is created within the projectile. Between these two magnetic fields an interaction is occurred [9].

The current that should pass through the projectile is given below when the distance taken by the $q$ load and the width of the projectile are taken into account [10].

$$
q=I_{t}=I \frac{l}{v_{d}} .
$$

Hence,

$$
\mathrm{d} q=I \mathrm{~d} t=I\left(\frac{\mathrm{d} x}{v_{d}}\right) .
$$

Here $I$ denote current and $l$ denotes the width of the projectile. When Eq. 3 is written into Eq. 2 and when the height of the projectile is examined in very tiny dimensions the following equation is attained.

$$
\mathrm{d} \boldsymbol{F}=\mathrm{d} q\left(\boldsymbol{v}_{d}\right) \boldsymbol{B}=\left(I \frac{\mathrm{d} x}{v_{d}}\right)\left(\boldsymbol{v}_{d}\right) \boldsymbol{B}=\boldsymbol{B} I \mathrm{~d} x .
$$

Equation 4 reveals the relationship between Lorentz forces those effects on the projectile according to the right-hand-rule with current and magnetic field. Besides, Biot-Savart law is utilized especially to solve the B magnetic field function [11].

Accordingly;

$$
B=\frac{\mu_{0} i}{4 \pi d} \text {. }
$$

Here, $\mu_{0} d$ enotes the magnetic permeability constant of air; $i$ denotes instantaneous current and $d$ denotes the distance from the conductor. For the calculation these two assumptions should also be made current flows thought the center of the rails, and the magnetic characteristics of the rectangular rails are the same with cylindrical conductors.
Here $x$ denotes the infinitely low diameter of the conductor and $R c$ denotes the diameter of the conductor. If Eq. 5 is rewritten in Eq. 4 and if the necessary integrations are taken, approximate Lorentz force between the rails are found as in the following equation.

$$
F=\frac{\mu_{0} I^{2}}{4 \pi} \ln \left[\frac{(r+l)^{2}}{r^{2}}\right] .
$$

Here, $r$ denotes the radius. The importance of Eq. 6 can be seen while defining Eq. 7. $L^{\prime}$ can be defined as inductance gradient and its unit is Henry/meter. Attention should be paid to the fact that the value of $L^{\prime}$ is not the inductance of the system. The value of $L^{\prime}$ may be defined as the magnetic field factor associated with the geometry of the electromagnetic weapon. Therefore, the value of $L^{\prime}$ in the design of electromagnetic weapon is constant.

$$
L_{,}=\frac{\mu_{0}}{2 \pi} \ln \left[\frac{(r+l)^{2}}{r^{2}}\right] .
$$

If Eq. 7 is rewritten in Eq. 6, Lorentz force can be simply written as follows,

$$
F=\frac{1}{2} L^{\prime} I^{2}
$$

Newton's second law is expressed as follows,

$$
F=m a \text {. }
$$

Accordingly, because of the assumption that current is constant along with the projectile, the acceleration equation necessary for the acceleration of the projectile is as follows,

$$
a=\frac{1}{2 m} L^{\prime} I^{2}=\frac{1}{2 m} L^{\prime}\left(\frac{Q}{t}\right)^{2} .
$$

In the equation $m$ denotes the mass of the projectile; $a$, the acceleration of the projectile; $Q$, load of the capacitors; and $t$, the duration of the flow of the load. Here, $Q$ load is transferred to the circuit, or to the projectile, by the capacitors in the duration $t$. In order to determine the speed of the projectile, the entrance of the projectile between the rails should be defined as $t=0$ while the exit of the projectile from the rails as $t=t$ and these values should be integrated to the above equation. Speed is the simple integral of acceleration. Accordingly,

$$
v=\int_{0}^{t} \frac{I^{2}}{2 m} L^{\prime} d t=\frac{L^{\prime}}{2 m} I Q=\frac{L^{\prime}}{2 m}\left(\frac{Q}{t}\right)^{2} t .
$$

Here, $v$ denotes speed. In the equation the values for the mass of the projectile and current pulse are assumed to be constant.

In order to make an analysis of the electromagnetic weapon, theoretically, the first step should be the assumption that the current has a constant value.

In practice, the system operates with instantaneous current that is subject to the values of capacitor and inductance. These factors may also be used to change the form of the current pulse. As can be observed in Fig. 1, the value of the current starts with zero and reaches its peak value with a sinusoidal trend, and decreases from peak value logarithmically. Accordingly, the increase of the current can be formulized [12]. 


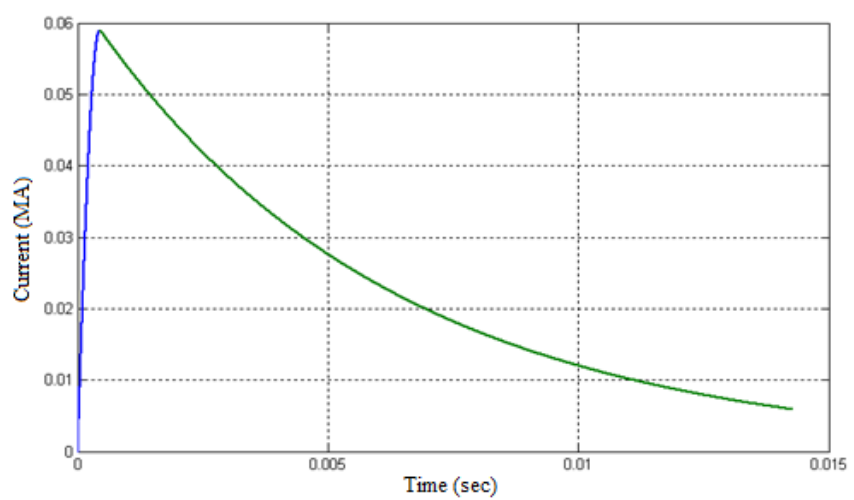

Fig. 1. Flow form of the current.

$$
I=I_{m} \sin (\omega t) .
$$

Here, $I_{m}$ and $\omega$ denote the peak value, and angular velocity of the current, respectively. The decrease of the current can be formalized as follows:

$$
I=I_{m} \mathrm{e}^{-\frac{R}{L} t} .
$$

Here $R$ denotes the voltage of the system and $L$ denotes the inductance of the system. The peak current equation is as follows [13, 14].

$$
I_{m}=V_{0} \sqrt{\frac{C}{L}} .
$$

In this equation $V_{0}$ and $C$ denote capacitor voltage and capacitor value, respectively. A projectile is desired to have minimum surface area in order it to resist high current density and this surface area is calculated according to maximum current density.

$$
A=\frac{I_{m}}{J_{\max }} .
$$

Here, $A$ denotes the surface area of the projectile and $J_{\max }$ denotes the maximum current density. In the study, maximum current density for instantaneous current is chosen as $2500 \mathrm{MA} / \mathrm{m}^{2}$.

The projectile volume is calculated with the multiplication of projectile surface area and the gap between rails, that is the width of the projectile. The following equation shows this calculation,

$$
H=A l \text {. }
$$

Here, $H$ denotes the volume of the projectile. The mass of the projectile is calculated with the multiplication of the density of the material used for the projectile and the volume of the projectile. The following Equation shows this multiplication,

$$
m=\rho H \text {. }
$$

Here, $\rho$ denotes the density of the projectile. In the study, by using the Eq. 16 and 17 the mass of the projectile used is found to be $3.05 \mathrm{gr}$.

As the equations are interconnected and multivariate a software program, namely Matlab, is utilized and the results and the graphs are prepared through Matlab.

The parameters and the necessary processes applied, and the results attained are given Table.
Parameters of rail type electromagnetic weapon.

\begin{tabular}{c|c}
\hline \hline Input values & Results \\
\hline$L^{\prime}=0.4321 \times 10^{-6}$ & $v=146.5 \mathrm{~m} / \mathrm{s}$ \\
$V_{0}=310 \mathrm{~V}$ & $I_{m}=59.04 \mathrm{KA}$ \\
$R=0.002 \Omega$ & $t_{1}=0.4487 \mathrm{~ms}$ \\
$L=1.5 \mu \mathrm{H}$ & $t_{2}=2.175 \mathrm{~ms}$ \\
$C=0.0544 \mathrm{~F}$ & Barrel: $22.76 \mathrm{~cm}$
\end{tabular}

If Fig. 2 is analyzed, it is seen that on the increasing edge of the current there is no much acceleration of the projectile however in the meanwhile the moment of inertia has been to a large extend overcome. In the decreasing edge of the current is seen that the projectile has gained the desired speed and left the barrel.

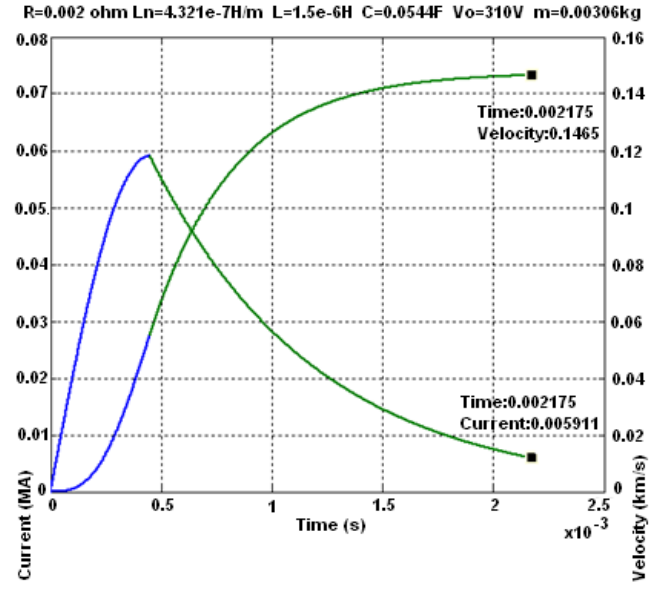

Fig. 2. The comparison of the graphs of the current pulse and speed.

\section{Production of rail electromagnetic weapon}

\subsection{Power sources}

Rail type electromagnetic weapons necessitate high levels of energy. In the project this energy is supplied through the capacitors. For the charging of the capacitors a direct current source is needed. In the project a bridge type rectifier is used.

At direct current as capacitors behave as if there is a short circuit at time zero they draw high current in the first instance. In order to prevent this inconvenience a strong resistor is attached to the output of the charging circuit.

\subsection{Capacitor and inductor}

In the system by establishing a $54400 \mu \mathrm{F}$, that is $0.054 \mathrm{~F}$, capacitor group by connecting 8 pieces of $6800 \mu \mathrm{F} 400 \mathrm{~V}$ capacitors in parallel, a high current source is supplied.

As known capacitors are charged or discharged at a logarithmic pace through a resistor. When an inductor is connected to the system in serially, it decelerates the 
velocity of fall and changes the form of current pulse. Hence, the duration of current to reach to zero is prolonged. By this means the effect of the current on the projectile would be increased. In the system, instead of a single $1.5 \mu \mathrm{H}$ inductor, two $0.75 \mu \mathrm{H}$ inductors are used and these inductors are located on the line from the capacitors to the rails.

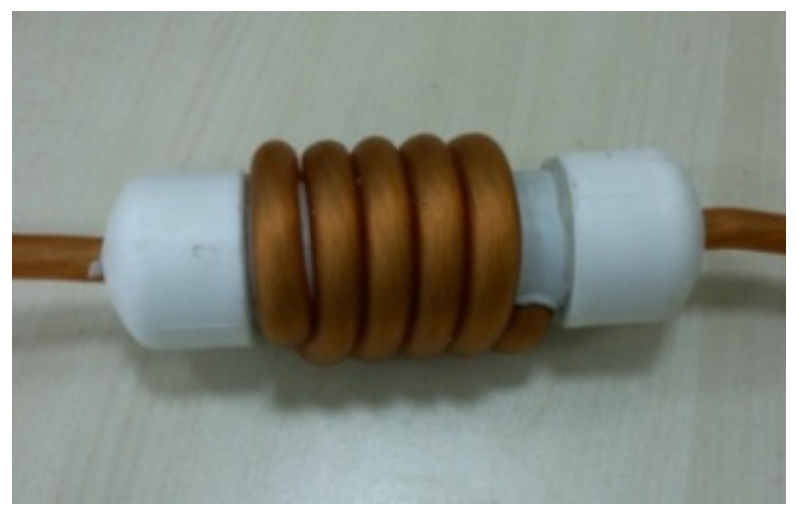

Fig. 3. Serial inductor.

\subsection{Air accelerator}

The first motion of the projectile in electromagnetic weapons is a big problem. In some systems, at first, the projectile is in between the rails. In such systems the energy is stored in the first stage, then by switching through mechanic or semi-conductor materials this energy stored is transmitted to the rails and projectile is fired. In the system, the air powered projectile repulsion technique is used. In order projectile to enter between the rails fast, high pressure air is needed. Therefore a compressed air reservoir is used in the system.

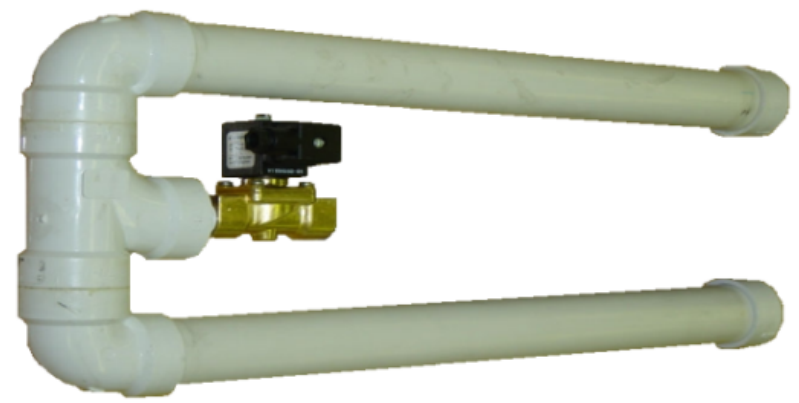

Fig. 4. Air accelerator.

\subsection{Barrel}

The most important part of electromagnetic weapons is the barrel. While the simplest way to adjust the influence setting is to adjust the quantity of energy stored, the shape of the barrel has also effect on the influence.

The major parts serving in the barrel are the rails. The rails should be produced from materials having high conductance and durability. The most ergonomic solution is copper rails. An alternative to decrease the friction and resistance of copper is to cover its surface with silver. These copper bars used must have profiles that can transfer the current without any warming.

As the currents passing through the rails are in opposite directions, the electromagnetic force created between the rails would repulse each other. Therefore the installation of Plexiglas rail grippers that would resist to this force into each other should be made efficiently.

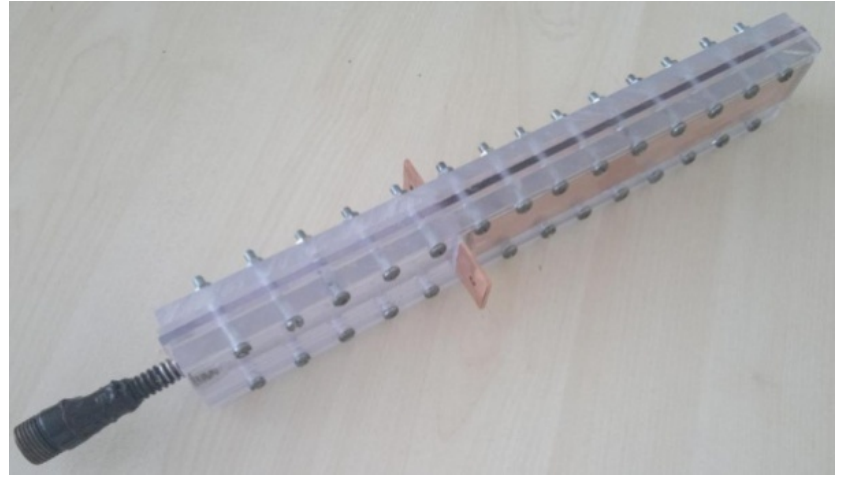

Fig. 5. The structure of barrel.

\subsection{The projectile}

One of the key points in the construction of an electromagnetic weapon is the projectile design and materials selection. The projectile is produced according to the weapon type.

All conductive materials may be used for projectile production but there are some inconveniences. For example, gold is a very good projectile material but it costs much. Copper and iron projectiles are economical but as they cause high levels of arc and deformation the pace of the projectile at the barrel decreases almost to zero. Being economical, high workability, low level of arc formation, low level of deformation and steady firing tests are the sufficient reasons for aluminum to be chosen as projectile material.

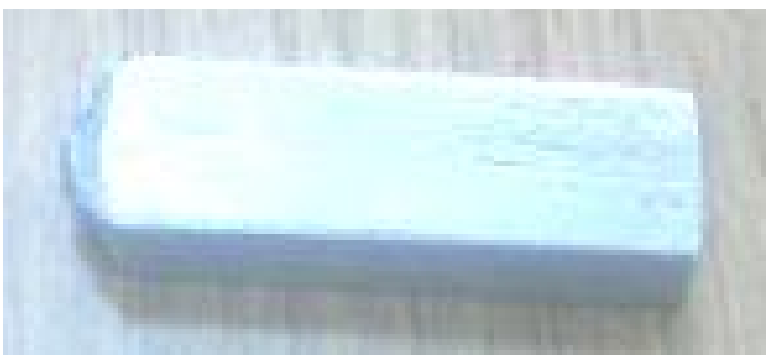

Fig. 6. The projectile used in the experiment.

\subsection{The installation of the components}

Except the aforementioned substances, some tiny parts are also utilized in the system. Connections are made as in Fig. 7 and the final version of the weapon that is ready to use is as in Fig. 8. 


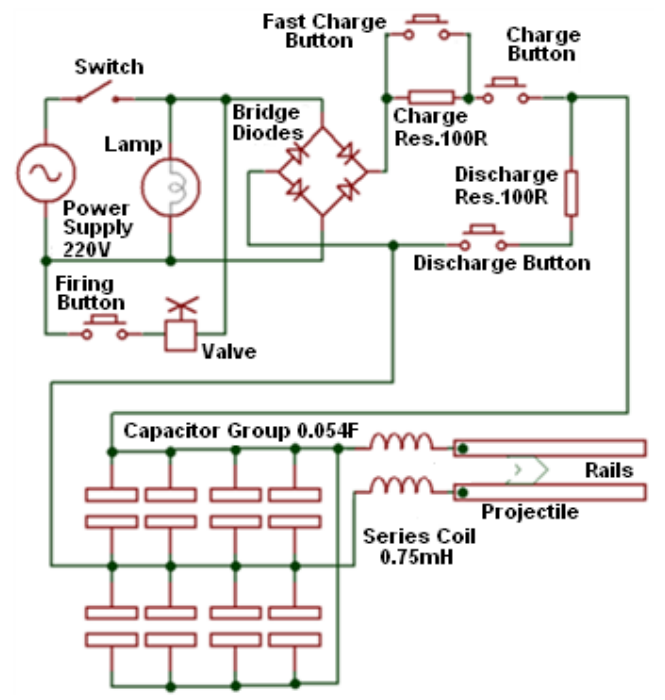

Fig. 7. Complete circuit of the system.

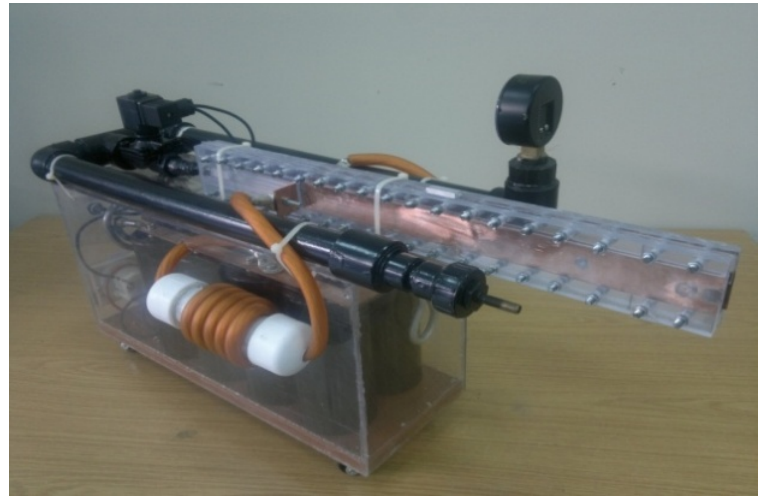

Fig. 8. Complete structure of the system.

\section{Results}

Although electromagnetic weapons seem quite simple logically, their calculations are not easy at all since a change in the system necessitates changes in other parameters. For example, when the projectile mass is desired to be changed when the speed is constant, capacity and the length of the barrel should also be changed. These calculations may be made with multivariate equations. In the study Matlab codes are prepared in order to make these calculations and present the results visually with graphs. Instead of solving quite a few equations, these codes are used and the desired values are entered to get the results.

In order to increase the speed of electromagnetic weapon the following three things should be realized:

-to increase the capacitor value

-to decrease the projectile mass

-to increase the voltage value

At the test shots no changes are made in the projectile mass or capacity value, instead by changing the value of voltage test are performed. Besides, some shots were unsuccessful and the projectiles got out of the barrel with low speeds. Further, during some shots the projectile clung to the rails and could not leave the rails. The results attained can be seen from the graph in Fig. 9.

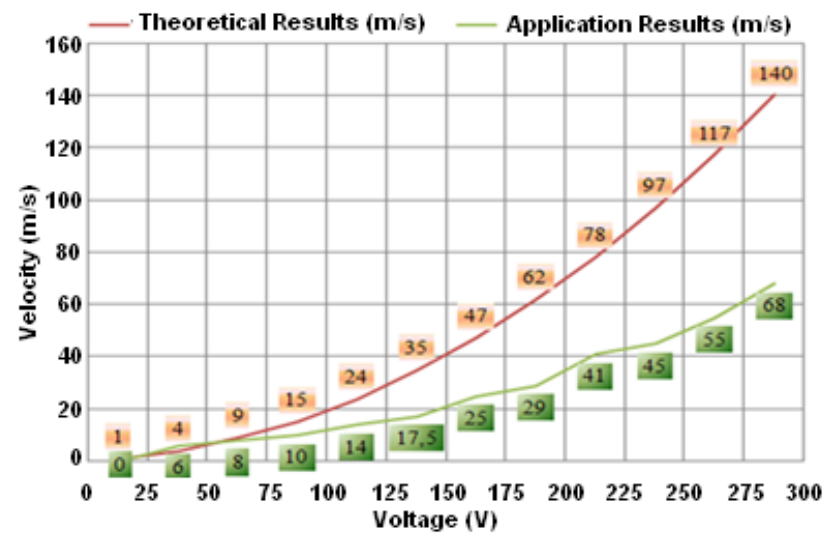

Fig. 9. Graph of voltage-speed relationship.

If the Graph is examined, it is seen that the application results and theoretical results of 12 test shots are compared.

It is evident that there is a huge gap between the speeds attained in practice and the ones in theory. The causes of this gap are the electrical losses, friction and especially the deformation at the rails and the projectile. With the melting due to the arc formed at the rails and projectile, projectile cling to the rails, chips pieces from the rails and speeds decrease because of increased friction. The deformation at the rails may be seen at the following Figure 10. As the melting point of aluminum is less than copper, the projectile melted more and the parts melted clung to the rails.

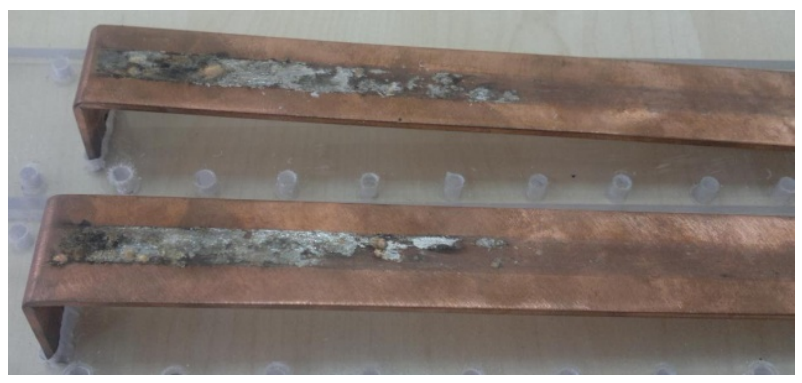

Fig. 10. The rails after the shot.

Consequently, a separate increase in the capacity is a solution for increasing the speed but it is not sufficient since an increase in the capacity causes increases energy losses and changes in the projectile mass. Accordingly, the type or shape changes because of its density. If these changes are not done, because of the arc there would be deformation at the projectile and rails.

Reductions in projectile mass are not an adequate solution itself to increase the speed. If this change is accommodated with an increase in the barrel length, the projectile would be projected without any energy loss. 
However, in this case, the disadvantage would be the contraction of the projectile, in other words a change in the material of the projectile and a decrease in the explosive material.

The method of increasing the voltage and speed adjustment is easier than changing the mass and the capacity. However, if a change in the length of the barrel would not be made in this method, waste energy arises, more arcs between the projectile and the rails are formed and hence more deformation at the rails occurs.

If the results are examined, it would be seen that the speed adjustment is a multivariate question. Instead of single variations in the voltage capacity or mass, it is healthier to adjust the speed with proper calculations and simultaneous changes.

The most practical way of attaining the ignition current is to use capacitors. However capacitors cannot be defined as complete power plants due both to their dimensions and capacities. The developments in the nuclear energy may help to the production of electromagnetic weapon systems that may be used in real life.

The current passing through a conductive causes it to warm up. As electromagnetic weapons extract high currents the system overheats. By applying necessary cooling techniques optimally and paying attention the choice of material to be used in the rails, precautions may be taken against warming.

Because of high temperature and current the rails may melt. Melting rails may change their shapes and their molecular structure may change. Attention should be paid to the atomic structure of the materials chosen.

It is necessary that the piece to be projected should transmit the current by contacting to both rails. If the projectile is too large, the speed may decrease and even it could not get out of the barrel. If the projectile is not large enough, it may not contact to both rails or due to the centrifugal motion it makes point contact, instead of superficial and the projectile would be projected without efficiency but with high deformation at the rails and the projectile itself. Therefore utmost attention should be paid to the dimensions of the projectile.

\section{Acknowledgments}

In this study, it was benefited from the master thesis named "Design and Experimental Analysis of Rail Type Electromagnetic Weapons System" in Electrical Education, Institute of Pure and Applied Sciences, Marmara University.

\section{References}

[1] A. Louis, Apparatus For Propelling Projectiles, United States Patent Office, 1421435, 1922.

[2] Ö. Akyazı, Fen ve Mühendislik Bilgisi Dergisi 20, 117 (2008).

[3] M. Başaydin, MSc Thesis, Gebze Yüksek Teknoloji Enstitüsü, 2009.
[4] S.C. Yurt, MSc Thesis, Istanbul Technical University, Graduate School of Science, Engineering and Technology, Istanbul, Turkey, 2011.

[5] A. Bolonkin, Journal of Aerospace Engineering, (2011).

[6] N. Pratikakis, Ph. D.Thesis, Naval Postgraduate School, TX, USA, 2006.

[7] C.C. Su, IEEE Transaction on Magnetics 42, (2006).

[8] A. Feliciano, PhD Thesis, Naval Postgraduate School, TX, USA, 2001.

[9] M.W. Smith, MSc Thesis, Naval Postgraduate School, TX, USA, 2002.

[10] İ.Y1ldı, MSc Thesis, Marmara Üniversitesi, Fen Bilimleri Enstitüsü, Y.Lisans Tezi, İstanbul, Türkiye, 2013.

[11] A.J. Edminister, in: Elektromanyetik, edited by $T$. Aydemir and E. Afacan and C. Nakiboğlu, Schaum's OutLines, Ankara, Turkey, 2000.

[12] D.S. Warnock, MSc Thesis, Naval Postgraduate School, TX, USA, 2003.

[11] P.M. Day, MSc Thesis, Texas Tech. University, TX, USA, 1992.

[14] L.Xu, Y. Geng, Applied Mathematical Modelling 36, 1465 (2012) 